\title{
On the spectral gap in Andreev graphs
}

\author{
Holger Flechsig and Sven Gnutzmann
}

\begin{abstract}
We introduce Andreev scattering (electron-hole conversion at an interface of a normal conductor to a superconductor) at the outer vertices of a quantum star graph and examine its effect on the spectrum. More specifically we show that the density of states in Andreev graphs is suppressed near the Fermi energy where a spectral gap may occur. The size and existence of such a gap depends on one side on the Andreev scattering amplitudes and, on the other side, on the properties of the electron-electron scattering matrix at the central vertex. We also show that the bond length fluctuations have a minor effect on the spectrum near the Fermi energy.
\end{abstract}

\section{Introduction and physical background}

The spectra of superconducting-normalconducting (SN) hybrid devices such as Andreev billiards has been a topic in physics for some time (see the recent review [1] for an overview and further references). An Andreev billiard consists of a finite normalconducting region with a boundary that is partly an interface to a superconductor. An electron with energy near the Fermi energy cannot propagate into the superconductor and it is trapped in a finite region similarly to a usual quantum billiard. However, the interface to the superconductor leads to an effective coupling of electron and hole dynamics through Andreev scattering. If an electron hits the interface to the superconductor it is reflected back into the billiard as a hole in the opposite direction of the incoming electron (this has been called "retroflection" to distinguish it from the specular reflection at an interface to an insulator). Physically the electron enters the superconductor where it excites an electron-hole pair. It then builds a Cooper pair with the electron while the hole is ejected back into the billiard. Quasiparticle excitations in such a device 
are described by the Bogoliubov-de Gennes equation

$$
\left(\begin{array}{cc}
-\nabla^{2}+V(\mathbf{x})-\mu & \Delta(\mathbf{x}) \\
\Delta(\mathbf{x}) & \nabla^{2}-V(\mathbf{x})+\mu
\end{array}\right) \boldsymbol{\psi}(\mathbf{x})=E \boldsymbol{\psi}(\mathbf{x}) .
$$

Here, $V(\mathbf{x})$ is a potential, $\mu \equiv k_{F}^{2}$ is the Fermi energy $\left(k_{F}\right.$ is the Fermi wave number), and $E$ the energy measured from the Fermi level. The wave function $\boldsymbol{\psi}(\mathbf{x})=\left(\begin{array}{l}u(\mathbf{x}) \\ v(\mathbf{x})\end{array}\right)$ has two components: $u(\mathbf{x})$ is the electron component and $v(\mathbf{x})$ the hole component. The electron and hole components are coupled by the pair potential $\Delta(\mathbf{x})$. The pair potential vanishes in a normalconducting region. In superconducting regions $\Delta(\mathbf{x}) \neq 0$ and waves can only propagate for $E>|\Delta(\mathbf{x})|$. In (1.1) we have assumed time-reversal invariance which means that there is no magnetic field and the pair potential is a real function. If $\boldsymbol{\psi}(\mathbf{x})=\left(\begin{array}{l}u(\mathbf{x}) \\ v(\mathbf{x})\end{array}\right)$ solves the Bogoliubov-de Gennes equation at energy $E$ then $\mathcal{C} \boldsymbol{\psi}(\mathbf{x})=\left(\begin{array}{c}-v(\mathbf{x})^{*} \\ u(\mathbf{x})^{*}\end{array}\right)$ is a solution to energy $-E$. This shows that the spectrum is symmetric around $E=0$ (that is around the Fermi energy) and reveals that these systems belong to one of the seven non-standard symmetry classes [2] (the three standard or Wigner-Dyson symmetry classes classify quantum systems according to their behaviour under time-reversal and spin rotation). The negative spectrum describes hole-like quasiparticle excitations (while the spectrum is negative, the physical energy of such an excitation is positive, of course) and the positive spectrum describes electron-like quasiparticle excitations.

It has been known for some time that Andreev reflections reduce the density of states near the Fermi energy and various universality classes have been defined $[\mathbf{2}, \mathbf{3}, 4, \mathbf{4}, \mathbf{6}, \mathbf{7}, \mathbf{8}, \mathbf{9}, \mathbf{1 0}]$ and related to quantum chaos. Spectral gaps have been found in irregularly shaped Andreev billiards for which the classical dynamics of the normal billiard (with specular reflection at the superconducting interface) is chaotic. Though Andreev scattering destroys chaos the Lyapunov exponent can be felt if the mean time $t_{A}$ between two subsequent Andreev reflections is large - the gap that was found is of the order $\hbar / t_{A}$ (in general much larger than the mean level spacing) [3, 4, 5]. In contrast an integrable normal billiard only leads to a suppressed density of states near the Fermi energy [7, 8]. Other universality classes such as magnetic billiards may have a chaotic dynamics even in the presence of Andreev reflections in that case the effect of Andreev scattering is universally described by 
appropriate Gaussian random-matrix ensembles [2, 10]. However the effect of Andreev scattering on the spectrum is less strong and decays after a few mean level spacings away from Fermi energy (which can only be seen after sufficiently averaging over system parameters).

Andreev scattering has been introduced to quantum maps [11 and quantum graphs [12]. The latter work investigated a class of Andreev graphs that corresponds to chaotic combined electron-hole dynamics with universal spectral statistics for non-standard symmetry classes. Chaos is however suppressed in a non-magnetic Andreev billiard. Here we will discuss the effect of Andreev reflections in a different class of graphs that is more closely related to non-magnetic Andreev billiards.

In Section 2 we will start our discussion with a simple model: a single lead with one interface to a superconductor at one end. This will serve as a motivation for our construction of Andreev graphs in Section 3.1. In the rest of Section 3 we will discuss the spectra of various ensembles of Andreev graphs - partly analytically, and numerically where analytical results could not be obtained straight forwardly.

\section{A hybrid SN lead}

Most of the concepts we will use later are most easily introduced for a one dimensional electronic hybrid SN lead.

2.1. Scattering from a SN interface. Let us start with discussing scattering from a SN interface at $x=0$. Let us assume that the pair potential is constant on the superconducting half line $x>0$ where $\Delta(x) \equiv \Delta_{0}$ while $\Delta(x) \equiv 0$ in the normalconducting half line $x<0$. At the interface we include a potential barrier $V(x)=V_{0} \delta(x)$. The scattering problem can be solved exactly for any set of parameters. We are interested in the case $E<\Delta_{0}$ in which there is no propagation in the superconducting half line. On the normalconducting half line a scattering solution of the Bogoliubov-de Gennes equation takes the form

$$
\boldsymbol{\psi}_{\mathrm{NC}}(x)=\left(\begin{array}{c}
a_{e, \mathrm{in}} \frac{e^{i k_{e} x}}{\sqrt{k_{e}}} \\
a_{h, \text { in }} \frac{e^{-i k_{h} x}}{\sqrt{k_{h}}}
\end{array}\right)+\left(\begin{array}{l}
a_{e, \text { out }} \frac{e^{-i k_{e} x}}{\sqrt{k_{e}}} \\
a_{h, \text { out }} \frac{e^{i k_{h} x}}{\sqrt{k_{h}}}
\end{array}\right)
$$

where $k_{e}=\sqrt{\mu+E}$ and $k_{h}=\sqrt{\mu-E}$ are the wave numbers of electrons and holes. Note that a hole propagating to the right (incoming wave) is described by $e^{-i k_{h} x}$. In (2.1) the outgoing coefficients $a_{e / h \text {,out }}$ 
are related to the incoming coefficients by the scattering matrix $S(E)$

$$
\left(\begin{array}{c}
a_{e, \text { out }} \\
a_{h, \text { out }}
\end{array}\right)=\left(\begin{array}{ll}
S_{e e}(E) & S_{e h}(E) \\
S_{h e}(E) & S_{h h}(E)
\end{array}\right)\left(\begin{array}{c}
a_{e, \text { in }} \\
a_{h, \text { in }}
\end{array}\right) .
$$

The scattering matrix can be obtained by matching the solution in the normalconducting part with a decaying solution in the superconducting part at $x=0$. The regime $E \ll \Delta_{0} \ll \mu=k_{F}^{2}$ will be relevant to us and simplifies the expressions considerably. Let us formalise this regime by using the observation that the difference of the electron wave number and the Fermi wave number is proportional to the energy $k_{e}-k_{F}=$ $\sqrt{k_{F}^{2}+E}-k_{F}=\frac{E}{2 k_{F}}+\mathcal{O}\left(E^{2} / k_{F}^{3}\right)$. Keeping this difference fixed as $k_{F} \rightarrow$ $\infty$ defines an interesting asymptotics. We thus introduce $\kappa=\frac{E}{2 k_{F}}$ as the new (rescaled) energy variable. All other system parameters have to be rescaled appropriately. For the pair potential we write $\Delta_{0}=k_{F}^{1+\alpha} \delta^{1-\alpha}$ where $\epsilon<\alpha<1-\epsilon$ which ensures $E \ll \Delta_{0} \ll \mu$. For the potential barrier $V_{0}=k_{F} v_{0}$ is the only way to scale the strength such that the resulting limiting scattering problem still contains a finite barrier. The leading order $S_{A}$ of the scattering matrix as $k_{F} \rightarrow \infty$ (keeping $\kappa, \delta$ and $v_{0}$ fixed) only depends on the potential barrier strength $v_{0}$, explicitly

$$
S_{A}=\left(\begin{array}{cc}
-\sqrt{1-t^{2}} e^{i \beta} & -i t \\
-i t & -\sqrt{1-t^{2}} e^{-i \beta}
\end{array}\right)
$$

where

$$
t=\frac{2}{2+v_{0}^{2}} \quad e^{i \beta}=\frac{v_{0}+2 i}{\sqrt{v_{0}^{2}+4}} .
$$

If there is no potential barrier $v_{0}=0$ the scattering matrix $S_{A}$ describes a pure Andreev reflection where an incoming electron (hole) is reflected as a hole (electron) and a phase factor $S_{h e} \rightarrow-i$ is acquired. In the opposite limit of a large potential barrier $v_{0} \rightarrow \infty$ one has pure electron-electron (hole-hole) scattering with $S_{e e}, S_{h h} \rightarrow-1$ which is equivalent to Dirichlet boundary conditions.

The leading asymptotics for the wave function (2.1) is obtained by replacing replacing $S \rightarrow S_{A}$ and $\frac{e^{ \pm i k_{e, h} x}}{\sqrt{k_{e, h}}} \rightarrow \frac{e^{ \pm i\left(k_{F} \pm \kappa\right) x}}{\sqrt{k_{F}}}$. Note that this contains fast oscillations $\propto e^{i k_{F} x}$. In the following we will always assume that $k_{F}$ is finite but large enough that it is justified to consider the leading asymptotic term only.

2.2. Excitation spectrum of a finite lead with one interface to a superconductor. We can now discuss the discrete excitation spectrum $\left\{\kappa_{n}=\frac{E_{n}}{2 k_{F}}\right\}$ of a finite normalconducting lead of length $L$ 
$(-L<x<0)$ which is attached to a superconductor at $x=0$ and to an insulator at the other end $x=-L$. We assume Dirichlet boundary conditions $\boldsymbol{\psi}_{\mathrm{NC}}(x=-L)=0$ at the interface to the insulator. Let us define the quantum map

$$
\mathcal{U}(\kappa)=S_{A} T(\kappa) S_{D} T(\kappa)
$$

where

$$
S_{D}=\left(\begin{array}{cc}
-1 & 0 \\
0 & -1
\end{array}\right) \quad \text { and } \quad T(\kappa)=\left(\begin{array}{cc}
e^{i\left(k_{F}+\kappa\right) L} & 0 \\
0 & e^{-i\left(k_{F}-\kappa\right) L}
\end{array}\right) .
$$

Here $T(\kappa)$ contains the phase factors acquired by the electron and hole components when propagated along the lead from one side to the other. The quantum map contains four steps: $i$. propagation from the superconducting interface to the insulating interface with $T(\kappa)$, ii. scattering from the insulator with $S_{D}$, iii. propagation back to the superconducting interface, and $i v$. scattering from the superconducting interface with $S_{A}$. If the quantum map $\mathcal{U}(\kappa)$ has an eigenvalue unity then $\kappa$ belongs to the excitation spectrum. The quantum map for the lead is periodic $\mathcal{U}\left(\kappa+\frac{\pi}{L}\right)=\mathcal{U}(\kappa)$ - the spectrum has the same periodicity and it suffices to know the first two positive eigenvalues $\kappa_{1}$ and $\kappa_{2}$. Explicitly, the quantum map is given by $\mathcal{U}(\kappa)=e^{i 2 L \kappa} \mathcal{U}_{0}$ with

$$
\mathcal{U}_{0}=\left(\begin{array}{cc}
\sqrt{1-t^{2}} e^{i\left(2 k_{F} L+\beta\right)} & i t e^{-i 2 k_{F} L} \\
i t e^{i 2 k_{F} L} & \sqrt{1-t^{2}} e^{\left.-i 2 k_{F} L+\beta\right)}
\end{array}\right)
$$

and has the eigenvalues

$$
u_{1}=e^{i(2 L \kappa-\phi)} \quad \text { and } \quad u_{2}=e^{i(2 L \kappa+\phi)}
$$

where

$$
\phi=\arccos \left(\cos \left(2 L k_{F}+\beta\right) \sqrt{1-t^{2}}\right) \in[0, \pi] .
$$

The first two positive eigenvalues in the spectrum of the lead are $\kappa_{1}=$ $\frac{\phi}{2 L}$ and $\kappa_{2}=\frac{2 \pi-\phi}{2 L}$ We arrive at the corresponding density of states

$$
d(\kappa)=\sum_{n=-\infty}^{\infty}\left[\delta\left(\kappa-\frac{2 \pi n+\phi}{2 L}\right)+\delta\left(\kappa-\frac{2 \pi n-\phi}{2 L}\right)\right] .
$$

We can now consider the mean density of states $\langle d(\kappa)\rangle_{k_{F}}$ averaged over different values of the Fermi wavelength $k_{F}$ where $\langle\odot\rangle_{k_{F}}=\lim _{K \rightarrow \infty} \frac{1}{K} \int_{k_{0}}^{k_{0}+K} \odot d k_{F}$. The result will only depend on the barrier strength $v_{0}$ or, equivalently, on the probability $p_{A}=t^{2}$ to be Andreev scattered at the superconducting interface. From (2.9) one observes that $\phi$ is always confined to the interval $\arcsin t \leq \phi \leq \pi-\arcsin t$ and every value in that interval 


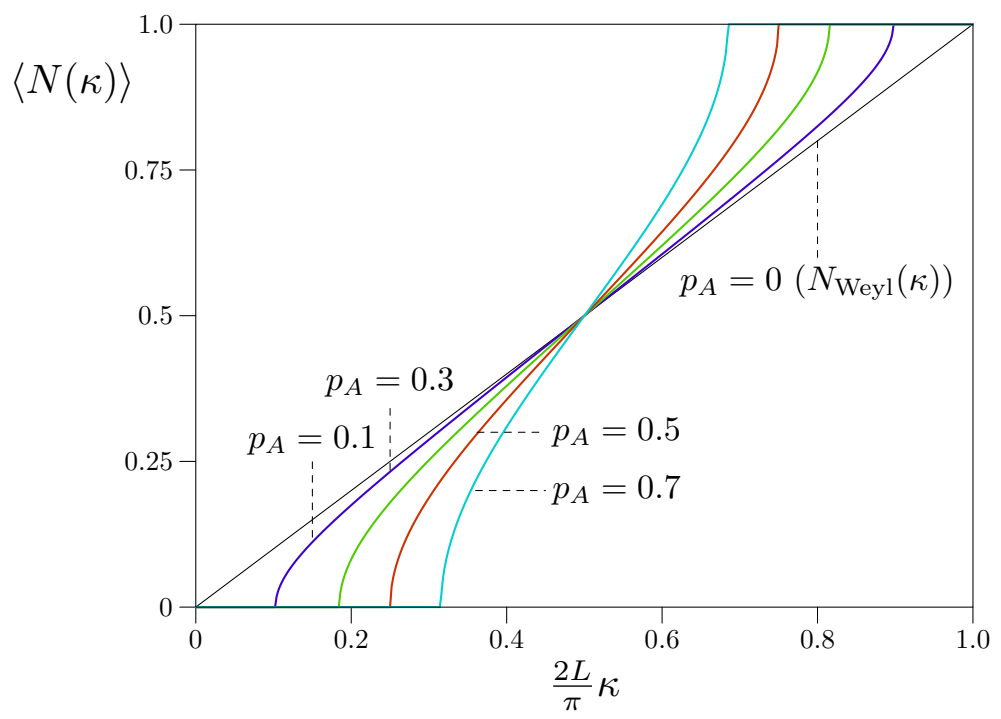

FiguRE 1. Averaged spectral counting function for a single lead (see text) for some values of the probability of Andreev scattering $p_{A}$. The same plots also apply to homogeneous Andreev star graphs with $B$ bonds and negligible bond length fluctuations (see Section 3.2.1).

is assumed for some $k_{F}$. As a consequence the mean density of states contains gaps of size $2 \Delta_{g}$ where

$$
\Delta_{g}=\frac{1}{2 L} \arcsin \sqrt{p_{A}}
$$

at $\kappa=n \pi(n=0, \pm 1, \pm 2, \ldots)$. The average over the Fermi wavelength $k_{F}$ can be performed straight forwardly by replacing $k_{F} L+\beta \rightarrow \theta$ and averaging over one period of $\theta$

$$
\begin{aligned}
\langle d(\kappa)\rangle_{k_{F}} & = \begin{cases}0 & \text { for } 0 \leq \kappa \leq \frac{\arcsin t}{2 L} \\
\frac{2 L}{\pi} \frac{\sin (2 L \kappa)}{\sqrt{\sin ^{2}(2 L \kappa)+t^{2}}} & \text { for } \frac{\arcsin t}{2 L} \leq \kappa \leq \frac{\pi-\arcsin t}{2 L} \\
0 & \text { for } \frac{\pi-\arcsin t}{2 L} \leq \kappa \leq \frac{\pi}{2 L}\end{cases} \\
\left\langle d\left(\kappa+\frac{\pi}{2 L}\right)\right\rangle_{k_{F}}=\langle d(\kappa)\rangle_{k_{F}} . &
\end{aligned}
$$


Figure 1 shows the corresponding averaged spectral counting function

$$
\begin{array}{rlrl}
\langle N(\kappa)\rangle_{k_{F}} & =\int_{0}^{\kappa} d \kappa^{\prime}\left\langle d\left(\kappa^{\prime}\right)\right\rangle_{k_{F}} & \\
& = \begin{cases}0 & \text { for } 0 \leq \kappa \leq \frac{\arcsin t}{2 L} \\
\frac{1}{\pi} \arccos \left(\frac{\cos (2 L \kappa)}{\sqrt{1-t^{2}}}\right) & \text { for } \frac{\arcsin t}{2 L} \leq \kappa \leq \frac{\pi-\arcsin t}{2 L} \\
1 & \text { for } \frac{\pi-\arcsin t}{2 L} \leq \kappa \leq \frac{\pi}{2 L}\end{cases} \\
\left\langle N\left(\kappa+\frac{\pi}{2 L}\right)\right\rangle_{k_{F}} & =\langle N(\kappa)\rangle_{k_{F}}+1 &
\end{array}
$$

for various values of the probability $p_{A}=t^{2}$ for Andreev scattering.

\section{The spectral gap in Andreev graphs}

In this chapter we will generalise the discussion of the lead with one superconducting/insulating interface at the ends to Andreev star graphs. A star graph $\mathcal{G}_{B}$ consists of one central vertex and $B$ outer (or peripheral) vertices which are connected to the centre by $B$ bonds (or edges). We assign a length $L_{b}(b=1, \ldots, B)$ and a position variable $x_{b} \in\left[0, L_{b}\right]$ to each bond. The star graph can then be quantised (and become a quantum star graph) by defining a Schrödinger operator on the graph (see [13, 14] and references therein). An Andreev star graph can similarly be constructed by defining a Bogoliubov-de Gennes operator on the graph.

Alternatively quantum graphs can be defined using the scattering formalism of Kottos and Smilansky [15. Their approach has been used to construct Andreev graphs [12] (and other classes of quantum graphs that contain particle-hole symmetries). We will follow the latter approach and assume that the peripheral vertices of the star graphs have an interface to a superconductor such that Andreev reflections can occur with a certain probability. The rest of the graph (the bonds and the central vertex) are normalconducting such that electron and hole components are not coupled. The construction takes care that the symmetry $\boldsymbol{\psi}(x)=\left(\begin{array}{l}u(x) \\ v(x)\end{array}\right) \mapsto \mathcal{C} \boldsymbol{\psi}(x)=\left(\begin{array}{c}-v(x)^{*} \\ u(x)^{*}\end{array}\right)$ which transforms positive energy states to negative energy states is also obeyed by the Andreev graph. Indeed the lead that we have discussed in the previous section is already an example of such an Andreev star graph with $B=1$. We will be interested what happens to the gap $\Delta_{g}$ for some 
ensembles of Andreev star graphs and how the properties of the density of states depend on the properties of the central vertex and the Andreev scattering amplitudes at superconducting vertices.

3.1. Construction of Andreev star graphs. Andreev star graphs can be quantised using a quantum map $\mathcal{U}(\kappa)$ in a similar way as we have treated the lead in the previous section. For a star graph with $B$ bonds the quantum map is a $2 B \times 2 B$ matrix of the form

$$
\mathcal{U}(\kappa)=S_{A} T(\kappa) S_{C} T(\kappa)
$$

where

$$
\begin{aligned}
T(\kappa) & =\left(\begin{array}{cc}
e^{i\left(k_{F}+\kappa\right) \mathcal{L}} & 0 \\
0 & e^{-i\left(k_{F}-\kappa\right) \mathcal{L}}
\end{array}\right) \\
\mathcal{L} & =\operatorname{diag}\left(L_{1}, \ldots, L_{B}\right)
\end{aligned}
$$

is a diagonal matrix which describes the propagation of an electron (first $B$ entries) or hole (last $B$ entries) from one end of each bond to the other $(\mathcal{L}$ is the diagonal $B \times B$ matrix which contains the lengths of the bonds), $S_{A}$ describes the scattering at the peripheral vertices and $S_{C}$ the scattering from the central vertex. The (partial) Andreev scattering at the peripheral vertices has four blocks

$$
S_{A}=\left(\begin{array}{cc}
-r & -i t \\
-i t & -r
\end{array}\right)
$$

which contain the two diagonal matrices

$$
\begin{aligned}
& t=\operatorname{diag}\left(t_{1}, \ldots, t_{B}\right) \\
& r=\operatorname{diag}\left(\sqrt{1-t_{1}^{2}}, \ldots, \sqrt{1-t_{B}^{2}}\right) .
\end{aligned}
$$

The real parameter $t_{b} \geq 0$ is the amplitude for Andreev scattering at the $b$-th vertex. We will not allow negative values for the Andreev amplitude here1. Comparing this to the scattering matrix at a superconducting interface in the previous section one may observe that we omitted the phase factor $e^{ \pm i \beta}$ in the electron-electron/hole-hole amplitude. These turn out to be irrelevant once we average the resulting spectra over the Fermi wave length (in the same way as they have been irrelevant for the lead - here we omit them from the start).

The bonds and the central vertex are assumed to be normalconducting. The electron-hole symmetry of the Bogoliubov-de Gennes equation

\footnotetext{
${ }^{1}$ Andreev graphs with mixed signs for the Andreev scattering amplitude belong to a different universality class [12 - in an Andreev billiard this would correspond to having two interfaces to different superconductors whose pair potentials have a relative phase $e^{-\pi}=-1$. No strong spectral gaps can be seen in such a regime.
} 
and time-reversal invariance then restrict the scattering matrix of the central vertex to the form [12]

$$
S_{C}=\left(\begin{array}{cc}
\sigma & 0 \\
0 & \sigma^{*}
\end{array}\right)
$$

where $\sigma$ is a symmetric unitary $B \times B$ matrix.

We will write $\mathcal{G}_{B}(\mathcal{U}(\kappa))$ for an Andreev star graph with $B$ bonds and quantum map $\mathcal{U}(\kappa)$. The spectrum of the Andreev star graph consists of the discrete (positive and negative) values $\kappa=\kappa_{n}$ for which the quantum map has an eigenvalue unity. Equivalently, the spectrum is given by the zeros of the characteristic equation

$$
\operatorname{spec}\left[\mathcal{G}_{B}(\mathcal{U}(\kappa))\right]=\left\{\kappa \in \mathbb{R}: \xi_{\mathcal{G}_{B}(\mathcal{U}(\kappa))}(\kappa, 1)=0\right\}
$$

where

$$
\xi_{\mathcal{G}_{B}(\mathcal{U}(\kappa))}(\kappa, \lambda)=\operatorname{det}(1-\lambda \mathcal{U}(\kappa))
$$

The spectrum (3.6) is symmetric around $\kappa=0-$ if $\kappa_{n}$ is in the spectrum so is $-\kappa_{n}$. This can be seen from the electron-hole symmetry

$$
\mathcal{U}(\kappa)^{*}=\left(\begin{array}{cc}
0 & 1 \\
-1 & 0
\end{array}\right) \mathcal{U}(-\kappa)\left(\begin{array}{cc}
0 & -1 \\
1 & 0
\end{array}\right)
$$

It will be convenient to define the density of states as

$$
d(\kappa)=\frac{1}{B} \sum_{n} \delta\left(\kappa-\kappa_{n}\right)
$$

which differs from the conventional form by the factor $1 / B$. Accordingly we define the spectral counting function

$$
N(\kappa)=\int_{0}^{\kappa} d\left(\kappa^{\prime}\right) d \kappa^{\prime}=\frac{1}{B} \sum_{\kappa_{n}>0} \Theta\left(\kappa-\kappa_{n}\right)
$$

(where we have assumed that $\kappa=0$ is not in the spectrum and $\Theta(x)$ is the Heaviside step function). The Kottos-Smilansky trace formula [15. expresses the spectral counting function (and hence the density of states) in terms of the characteristic equation

$$
\begin{aligned}
N(\kappa) & =N_{\text {Weyl }}(\kappa)+N_{\text {osc }}(\kappa) \\
N_{\text {Weyl }}(\kappa) & =\frac{2 \operatorname{tr}(\mathcal{L})}{B \pi} \kappa \\
N_{\text {osc }}(\kappa) & =-\frac{1}{\pi B} \lim _{\lambda \rightarrow 1^{-}} \operatorname{Im} \ln \xi_{\mathcal{G}_{B}(\mathcal{U}(\kappa))}(\kappa, \lambda) .
\end{aligned}
$$

The contribution $N_{\text {osc }}(\kappa)$ is an oscillating function of the spectral parameter $\kappa$ while $N_{\text {Weyl }}(\kappa)$ describes the mean increase of the number of states over a large interval of the (rescaled) energy $\kappa$. The oscillating 
part of the counting function does not necessarily vanish if averaged over some system parameter at fixed $\kappa$.

3.2. Andreev star graphs with negligible bond length fluctuations. Let us introduce the bond length fluctuations by writing

$$
L_{b}=L+\delta L_{b} \quad \text { where } \sum_{b} \delta L_{b}=0
$$

where $L$ is the mean bond length. If the fluctuations $\delta L_{b}$ are very small, and for small enough $\kappa$ one has $e^{i L_{b} \kappa} \approx e^{i L \kappa}$. Indeed we will only be interested in the spectrum on a scale $|\kappa| \lesssim C \frac{\pi}{2 L}$ (such that $N_{\text {Weyl }}(\kappa) \lesssim C$ and $C$ is of order unity) and assuming $\delta L_{b} \frac{\pi}{2 L} \ll 1 / C$ we will set $e^{i \delta L_{b} \kappa} \mapsto 1$. As a consequence $\mathcal{U}\left(\kappa+\frac{\pi}{L}\right)=\mathcal{U}(\kappa)$ is periodic (and hence is the spectrum). Also, the spectrum and eigenfunctions of the Andreev graph can be constructed straight forwardly from the $2 B$ eigenvalues and eigenvectors of the quantum map $\mathcal{U}(\kappa)$ at $\kappa=0$.

The small fluctuations in the bond lengths can however not be neglected in the phase factors containing the Fermi wavenumber $k_{F}$-indeed we want to average spectral functions over arbitrary large values of the Fermi wavenumber. A further significant simplification is obtained by assuming that all bond length are rationally independent. By setting $e^{i k_{F} L_{b}} \mapsto e^{i \theta_{b}}$ we may then replace an average over the Fermi wavelength by an average over a $B$-torus

$$
\begin{aligned}
\left\langle\mathcal{F}\left(e^{i k_{F} L_{1}}, \ldots, e^{i k_{F} L_{B}}\right)\right\rangle_{k_{F}} & =\left\langle\mathcal{F}\left(e^{i \theta_{1}}, \ldots, e^{i \theta_{B}}\right)\right\rangle_{\theta} \\
& =\frac{1}{(2 \pi)^{B}} \int d^{B} \theta \mathcal{F}\left(e^{i \theta_{1}}, \ldots, e^{i \theta_{B}}\right) .
\end{aligned}
$$

Writing $\boldsymbol{\theta}=\operatorname{diag}\left(\theta_{1}, \ldots, \theta_{B}\right)$ we arrive at the following class of ensembles of quantum maps for an Andreev star graph with $B$ bonds and negligible bond length fluctuations

$$
\begin{aligned}
\mathcal{U}(\kappa) & =\left(\begin{array}{cc}
-r & -i t \\
-i t & -r
\end{array}\right)\left(\begin{array}{cc}
e^{i \boldsymbol{\theta}} e^{i L \kappa} & 0 \\
0 & e^{-i \boldsymbol{\theta}} e^{i L \kappa}
\end{array}\right)\left(\begin{array}{cc}
\sigma & 0 \\
0 & \sigma^{*}
\end{array}\right)\left(\begin{array}{cc}
e^{i \boldsymbol{\theta}} e^{i L \kappa} & 0 \\
0 & e^{-i \boldsymbol{\theta}} e^{i L \kappa}
\end{array}\right) \\
& =-e^{i 2 L \kappa}\left(\begin{array}{cc}
r & i t \\
i t & r
\end{array}\right)\left(\begin{array}{cc}
e^{i \boldsymbol{\theta}} \sigma e^{i \boldsymbol{\theta}} & 0 \\
0 & e^{-i \boldsymbol{\theta}} \sigma^{*} e^{-i \boldsymbol{\theta}}
\end{array}\right)
\end{aligned}
$$

where $\theta_{1}, \ldots, \theta_{B}$ are $B$ independent random variables that are uniformly distributed over the interval $[0,2 \pi)$. The remaining parameters such as the central electron-electron scattering matrix $\sigma$ and the Andreev scattering amplitudes $t_{b}$ are assumed to be fixed in one ensemble. We will call the ensemble homogeneous if all Andreev scattering amplitudes are equal $t_{b}=t$, otherwise we call it inhomogeneous. We 
will now solve the homogeneous case and discuss some inhomogeneous ensembles numerically.

3.2.1. The homogeneous ensemble. In this case the ensemble averaged spectral counting will turn out to be completely independent of the choice of the central electron-electron scattering matrix, and also of the number of bonds. In fact the case can be reduced to the case $B=1$ that we have already solved. To see this we write $\theta_{b}=\theta+\delta \theta_{b}$ such that $\sum \delta \theta_{b}=0$. We will first do the average over $\theta$ and observe that this can be solved by diagonalisation. The straight forward result of the average over $\theta$ will not depend on $\delta \theta_{b}$.

To do the average over $\theta$ let us fix $\boldsymbol{\delta} \boldsymbol{\theta}$ and diagonalise

$$
e^{i \boldsymbol{\delta} \boldsymbol{\theta}} \sigma e^{i \boldsymbol{\delta} \boldsymbol{\theta}}=O e^{i \boldsymbol{\nu}} O^{T}
$$

where $O$ is a real orthogonal matrix (remember that $\sigma$ is symmetric) and $e^{i \boldsymbol{\nu}}=\operatorname{diag}\left(e^{i \nu_{1}}, \ldots, e^{i \nu_{B}}\right)$ contains the unimodular eigenvalues on the diagonal (we will omit the explicit dependence of the orthogonal matrix and the eigenvalues on $\delta \theta_{b}$ ). We may now write

$$
\mathcal{U}(\kappa)=\left(\begin{array}{ll}
O & 0 \\
0 & O
\end{array}\right)\left(\begin{array}{cc}
r & i t \\
i t & r
\end{array}\right)\left(\begin{array}{cc}
e^{i(2 \theta+2 \kappa L+\pi+\boldsymbol{\nu})} & 0 \\
0 & e^{-i(2 \theta-2 \kappa L+\pi+\boldsymbol{\nu})}
\end{array}\right)\left(\begin{array}{cc}
O^{T} & 0 \\
0 & O^{T}
\end{array}\right)
$$

and observe that the spectral counting function can be written as a sum over $B$ modes each of which is equivalent to the single lead (the case $B=1$ ). As a consequence the position and size of the spectral gaps are the same as for the single lead and the averaged density of states and the averaged spectral counting function are described by (2.12) and (2.13) respectively (any dependence on the $\delta \theta_{b}$ has dropped). It is remarkable that this result is universal - it only depends on the Andreev scattering amplitude and on no other system parameter (the dependence on the length $L$ is trivial).

3.2.2. Some inhomogeneous ensembles. Different Andreev scattering amplitudes at the peripheral vertices introduce an additional amount of disorder in the inhomogeneous case. One may expect that the form of the averaged density of states now depends on the actual choice of amplitudes $t_{b}$ and on the central electron-electron scattering matrix $\sigma$ - and the same applies to the existence and size of spectral gaps. In this section we will only discuss the extreme case $t_{b} \in\{0,1\}$ - that is, at some peripheral vertex one has either complete Andreev reflections $t_{b}=1$ or complete normal reflections $t_{b}=0$. Let $M \leq B$ be the number of bonds with total Andreev reflection such that $t_{b}=0$ for $b=1, \ldots B-M$ and $t_{b}=1$ for $b=B-M+1, \ldots, B$. The mean probability to be Andreev scattered is $p_{A}=\frac{M}{B}$. 
One consequence of our choice of Andreev scattering amplitudes is that averaging over the phases $\theta_{B-M+1}, \ldots, \theta_{B}$ is completely irrelevant. To see this let us assume an electron is scattered into one of the bonds $b=B-M+1, \ldots, B$. It accumulates a phase $e^{i\left(\theta_{b}+\kappa L\right)}$ as it propagates from the centre to the peripheral vertex where it is scattered back as a hole and acquires a further phase factor $-i$. When the hole has propagated back to the centre the overall phase which has been accumulated is $-i e^{2 \kappa L}$ where the contribution from the random phase $\theta_{b}$ has been cancelled exactly.

One further implication can be drawn from looking at the eigenvectors of the matrix $\tilde{\sigma}(\boldsymbol{\theta})=e^{i \boldsymbol{\theta}} \sigma e^{i \boldsymbol{\theta}}$ where $\boldsymbol{\theta}=\operatorname{diag}\left(\theta_{1}, \ldots, \theta_{B}\right)$ is fixed. In general it is not possible to construct eigenstates of the Andreev star graph from the eigenvectors $\tilde{\sigma}$ because these get coupled in a different way at normal reflecting and at Andreev reflecting peripheral vertices. However, if $\tilde{\sigma}$ has an eigenvector $\mathbf{a}=\left(0, \ldots, 0, a_{B-M+1}, \ldots, a_{B}\right)^{T}$ with eigenvalue $e^{i \alpha}$ which is supported completely on the bonds $b=$ $B-M+1, \ldots, B$ then it is straight forward to show that $\mathcal{U}(0)$ has the two eigenstates $\left(\begin{array}{c}e^{-i \alpha / 2} \mathbf{a} \\ \pm e^{i \alpha / 2} \mathbf{a}\end{array}\right)$ which are also supported on the bonds $b=B-M+1, \ldots, B$. The corresponding eigenvalue is $\mp i$ which contributes to the spectrum of the Andreev graph at $\kappa \frac{2 L}{\pi}=n+\frac{1}{2}(n$ integer). Furthermore, if there is such a state for some fixed choice of $\boldsymbol{\theta}$ then there will be such a state for any element in the ensemble and one will observe a corresponding step in the averaged counting function. A similar statement does not hold for states that are supported on the normalconducting bonds $b=1, \ldots, B-M$.

In the following we will restrict our discussion to two choices for the central electron-electron scattering matrix that are very well studied in the context of normal quantum graphs:

$i$. the Neumann (or Kirchhoff) scattering matrix

$$
\sigma_{\mathrm{Neumann} b b^{\prime}}=\frac{2}{B}-\delta_{b b^{\prime}}
$$

and $i$. the discrete Fourier transform (DFT) matrix

$$
\sigma_{\mathrm{DFT} b b^{\prime}}=\frac{1}{\sqrt{B}} e^{i 2 \pi \frac{b b^{\prime}}{B}}
$$

For quantum star graphs (with a scalar wave function and with incommensurable bond lengths) it is known that the DFT matrix generates universal spectral statistics as known from quantum chaotic systems 


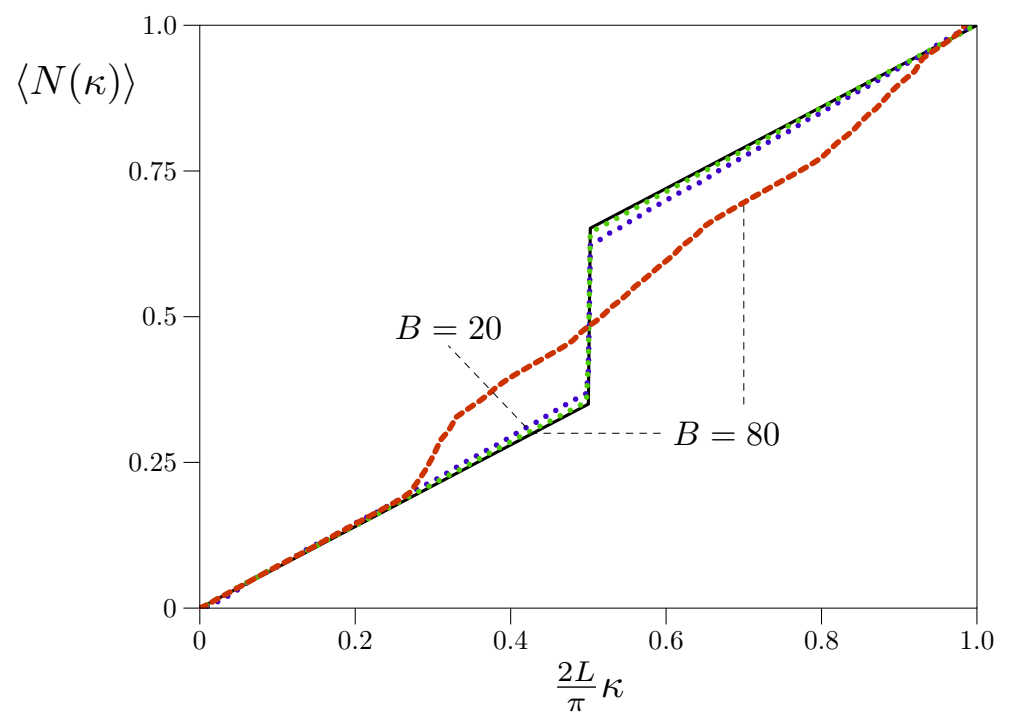

FiguRE 2. Dotted and full lines: averaged spectral counting function for Andreev graphs with negligible bond length fluctuations, inhomogeneous Andreev scattering, and a Neumann e-e scattering matrix. The two dotted lines are for $B=20, M=6$ (blue) and for $B=80, M=24$ (green) such that $p_{A}=0.3$ in both cases. The full (black) line is the expected limiting function and hardly differs from the line for $B=80$.

Dashed (red) line: averaged spectral counting function for the same inhomogeneous ensemble but with one set of randomly chosen bond lengths.

and the Gaussian orthogonal ensemble (GOE) of random matrix theory. In contrast, the Neumann star graph belongs to a different universality class with intermediate spectral statistics.

We first discuss the inhomogeneous ensemble with a Neumann e-e scattering matrix $\sigma_{\text {Neumann }}$. The spectral decomposition of $\sigma_{\text {Neumann }}$ is straight forward. It has one eigenvalue 1 and $B-1$ eigenvalues -1 . For our discussion it is important that one can construct $M-1$ orthogonal eigenvectors of $\sigma_{\text {Neumann }}$ that are supported on the bonds $b=B-M+1, \ldots, B$. According to our discussion above this leads to $2(M-1)$ eigenvectors of the quantum map $\mathcal{U}(0)$ with eigenvalues $\pm i$ and the averaged spectral counting function will have steps of size $\frac{M-1}{B}=p_{A}-\frac{1}{B}$ at $\kappa \frac{2 L}{\pi}= \pm \frac{1}{2}+n(n=0, \pm 1, \pm 2, \ldots)$. On each of these modes the probability to be Andreev scattered is unity. The remaining 
$2(B-M+1)$ modes are hardly coupled (at least for large $B)$ by Andreev scattering - the mean probability to be Andreev scattered among these modes is of the order $\frac{1}{B}$ and we may expect that the effect on these modes is negligible in the limit $B \rightarrow \infty$ (where $p_{A}=\frac{M}{B}$ is fixed) such that the contribution to the averaged spectral counting function is linear. Figure 2 compares the numerically obtained averaged counting function for an Andreev graph with a Neumann e-e scattering matrix with the expected limiting function

$$
N(\kappa)_{\text {Neumann }}=\left(1-p_{A}\right) \kappa \frac{2 L}{\pi}+\sum_{n=-\infty}^{\infty} p_{a} \Theta\left(\kappa \frac{2 L}{\pi}-\frac{1}{2}-n\right)
$$

Our expectation seems to be confirmed by the numerics and suggests that while there remains no gap in the averaged density of states the latter is reduced compared to Weyl's law near $\kappa=0$ (modulo periodicity).

Let us now look more closely at the spectrum near $\kappa=0$ for a finite size of the graph. Figure 3 shows the integrated distribution $I_{1}(\kappa)$ of the first positive eigenvalue of the Andreev graph as a function of $\frac{2 L B}{\pi} \kappa$ (that is we measure the spectrum in units of the mean level spacing according to Weyl's law). The numerics suggests that even on this blown-up scale there is no gap. $I_{1}(\kappa)$ may either start algebraically $I_{1} \sim \kappa^{\gamma}$ or as a power series. In both cases it implies that there is a further reduction (in addition to the one discussed above) of the density of states on this blown-up scale. We used 1000 realisations in our numerics which is not sufficient to resolve the region $\frac{2 L B}{\pi} \kappa<0.01$ so our results are not fully conclusive.

The behaviour of the spectral counting function and $I_{1}(\kappa)$ is very different if we choose the DFT e-e scattering matrix. Figure 4 shows the numerically averaged spectral counting function for this case. There are several interesting features in this spectrum. Again, we find a jump at $\frac{2 L}{\pi} \kappa=\frac{1}{2}$ which is connected to eigenvectors of $\sigma_{\text {DFT }}$ which are supported on the bonds $b=B-M+1, \ldots, B$ where the Andreev scattering occurs. However, the step is much smaller compared to the Neumann case and the probability for Andreev scattering on the remaining modes is large enough to have an effect. For large $B$ the spectral counting function converges (numerically) to a limiting function - the plots in Figure 4 are for $B=80$ and hardly differ from the case $B=40$ (not shown). Most importantly the counting function seems to drop to zero at a finite positive value of $\kappa$.

The numerically obtained distribution of the first eigenvalue $I_{1}(\kappa)$ in 


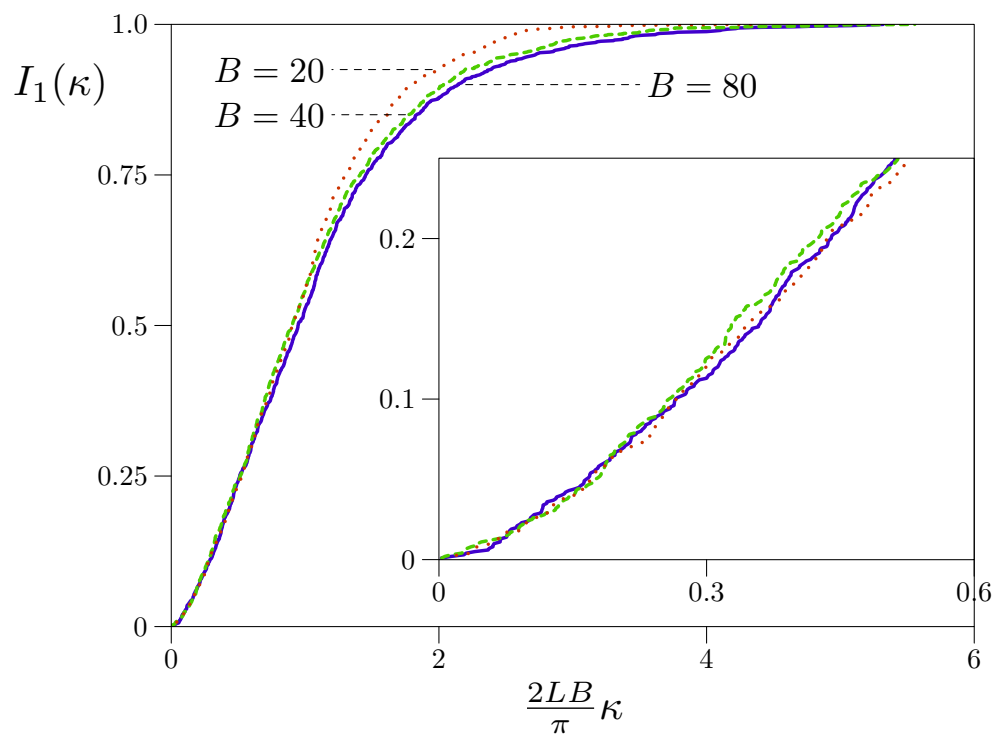

FIGURE 3. Integrated Distribution of the first positive eigenvalue of three Andreev star graphs with negligible bond length fluctuations, inhomogeneous Andreev scattering $\left(p_{A}=0.3\right.$ for all curves) and Neumann e-e scattering matrix. The three plots correspond to an increasing number of bonds $(B=20,40,80)$.

Figure 5 reveals that a spectral gap appears at least in the limit $B \rightarrow \infty$ (at constant $p_{A}$ ).

3.3. Andreev star graphs with random bond lengths. Let us now discuss the influence of bond lengths and discuss ensembles of Andreev graphs for which the bond length fluctuations cannot be ignored. For a graph with $B$ bonds let us choose a fixed set of bond lengths in the interval $0<L_{b}<1$ and now average over the Fermi wave number $k_{F}$. The main effect of the different bond lengths is that one cannot get the spectrum of the graph from just diagonalising the quantum map at $\kappa=0$ and the spectral properties are no longer periodic. In fact one should expect that any features in the counting function which differ from Weyl's law can only survive near $\kappa=0$.

We have calculated the averaged counting function numerically using the trace formula (3.11). We chose the bond lengths independently in the interval $0 \leq L_{b} \leq 1$ with a random number generator. All plots we show are for a single realisation of the bond lengths (we have assured that the figures are generic by comparing to other choices). Indeed Figures 2 and 4 show that the averaged counting function for both 


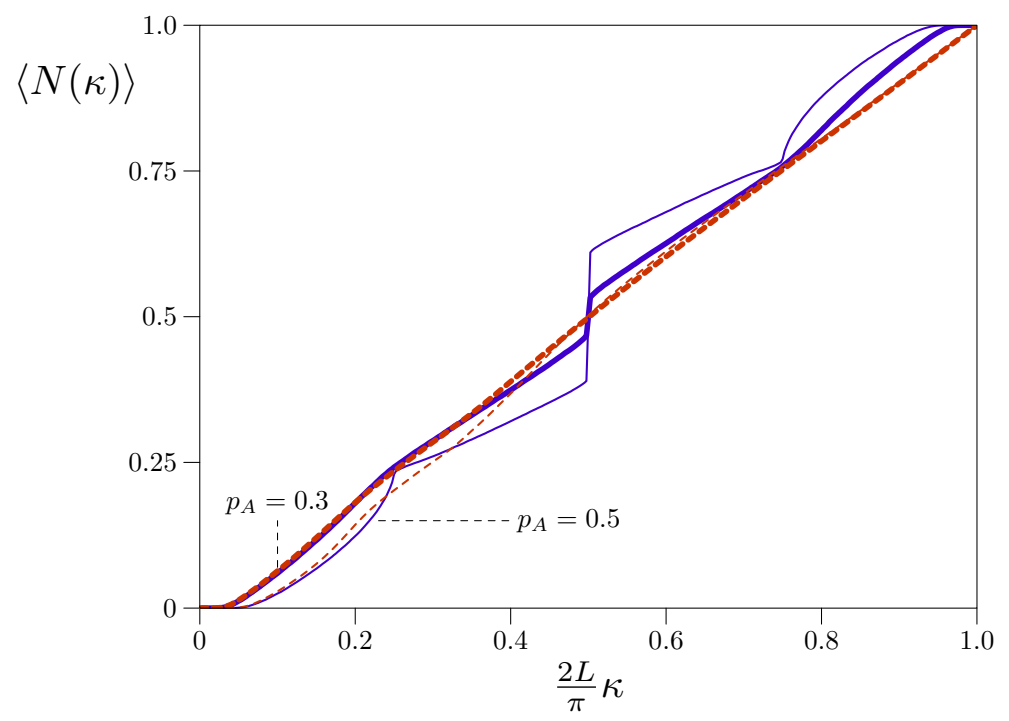

Figure 4. Full (blue) lines: averaged spectral counting function for Andreev graphs with negligible bond length fluctuations, inhomogeneous Andreev scattering, and a DFT e-e scattering matrix. The fat line corresponds to $B=80, M=24\left(p_{A}=0.3\right)$ and the thin line to $B=80$, $M=40\left(p_{A}=0.5\right)$. Plots for $B=40$ and the same values for $p_{A}$ (not shown) are almost identical.

Dashed (red) lines: averaged spectral counting function for the same inhomogeneous ensemble but with one set of randomly chosen bond lengths.

the Neumann and the DFT e-e scattering matrix has lost all special features when $\frac{2 L}{\pi} \kappa$ is on the order of one. For smaller values $\frac{2 L}{\pi} \kappa \ll 1$ the fluctuations in the bond lengths are not effective and one gets back to the case discussed before. In short, the bond length fluctuations destroy some special features of the previous ensembles with negligible bond length fluctuations. The bond length fluctuations do not have a strong influence on the reduction of the density of states near $\kappa=0$ or the existence and size of a gap, at least when $p_{A} \ll 12$.

\section{Conclusions}

We have shown that the spectra of Andreev star graphs have a variety of special spectral properties that are related to the introduction of Andreev scattering into a quantum graph. There is a spectral gap in

\footnotetext{
${ }^{2}$ This should be taken with care since in our model the bond length fluctuation are still moderate.
} 


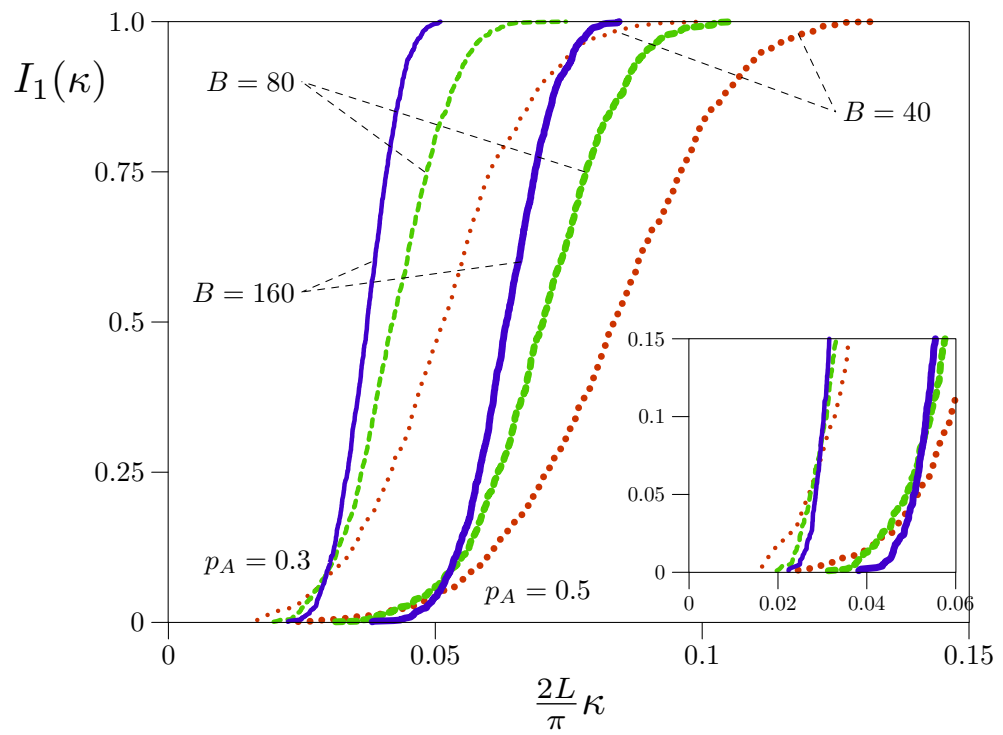

FiguRE 5. Integrated Distribution of the first positive eigenvalue of Andreev star graphs with negligible bond length fluctuations, inhomogeneous Andreev scattering and DFT e-e scattering matrix. The three plots on the left correspond to $p_{A}=0.3$, the three on the right to $p_{A}=0.5$. In both cases we have plotted the distribution for $B=40$ (dotted red lines), $B=80$ (dashed green lines), and $B=160$ (full blue lines).

homogeneous ensembles of Andreev graphs. Both the spectral counting function and the spectral gap are universal and only depend on the probability of Andreev scattering $p_{A}$.

Inhomogeneous ensembles contain additional disorder and the universal results from the homogeneous case are not applicable. Instead there is a rich variety of phenomena that depend on the properties of the central scattering matrix. For some special choices we could show numerically that the spectral counting functions converge to a limiting function as $B \rightarrow \infty$.

This first numerical investigation indicates that the appearance and size of a spectral gap is strongly related to the localisation properties of the eigenstates of the central e-e scattering matrix.

\section{References}

[1] C.W.J Beenakker, Lect. Notes Phys. 667, 131 (2005).

[2] A. Altland and M.R. Zirnbauer, Phys. Rev. Lett. 76, 3420 (1996); Phys. Rev. B 55, 1142 (1997). 
[3] J.A. Melsen, P.W. Brouwer, K.M. Frahm, and C.W.J. Beenakker, Europhys. Lett. 35, 7 (1996).

[4] K.M. Frahm, P.W. Brouwer, J.A. Melsen, and C.W.J. Beenakker, Phys. Rev. Lett. 76, 2981 (1996).

[5] A. Lodder and Yu.V. Nazarov, Phys. Rev. B 58, 5783 (1998).

[6] J. Wiersig, Phys. Rev. E 65, 036221 (2002)

[7] J. Cserti, A. Kormanyos, Z. Kaufmann, J. Koltai, and C.J. Lambert, Phys. Rev. Lett. 89, 057001 (2002).

[8] J. Cserti, A. Bodor, J. Koltai, and G. Vattay, Phys. Rev. B 66, 064528 (2002).

[9] A. Ossipov and T. Kottos, Phys. Rev. Lett. 92, 017004 (2004).

[10] S. Gnutzmann, B. Seif, F. von Oppen, and M.R. Zirnbauer, Phys. Rev. E 67, 046225 (2003).

[11] Ph. Jacquod, H. Schomerus, and C.W.J. Beenakker, Phys. Rev. Lett. 90, 207004 (2003).

[12] S. Gnutzmann and B. Seif Phys. Rev. E 69056219 (2004); Phys. Rev. E 69 056220 (2004).

[13] P. Kuchment, Waves Random Media 12, R1 (2002).

[14] S. Gnutzmann and U. Smilansky, Advances in Physics, 55, 527 (2007).

[15] T. Kottos and U. Smilansky, Phys. Rev. Lett. 79, 4794 (1997); Ann. Phys. 274, 76 (1999).

Fachbereich Physik, Freie Universität Berlin, Germany

Current address: Department of Physical Chemistry, Fritz-Haber-Institute of the Max Planck Society, Berlin, Germany

E-mail address: flechsig@fhi-berlin.mpg.de

School of Mathematical Sciences, University of Nottingham, United KINGDOM

E-mail address: sven.gnutzmann@nottingham.ac.uk 